\title{
PET Imaging of Colorectal and Breast Cancer by Targeting EphB4 Receptor with ${ }^{64} \mathrm{Cu}-L a b e l e d ~ h A b 47$ and hAb131 Antibodies
}

\author{
Shuanglong Liu*1, Dan Li*1,2, Ryan Park ${ }^{1}$, Ren $\mathrm{Liu}^{3}$, Zanxian Xia ${ }^{4}$, Jiacong Guo ${ }^{1}$, Valery Krasnoperov ${ }^{4}$, Parkash S. Gill ${ }^{3}$, \\ Zibo $\mathrm{Li}^{1}$, Hong Shan ${ }^{2}$, and Peter S. Conti ${ }^{1}$ \\ ${ }^{I}$ Molecular Imaging Center, Department of Radiology, University of Southern California, Los Angeles, California; ${ }^{2}$ Department of \\ Radiology, Third Affiliated Hospital of Sun Yat-Sen University, Guangzhou, China; ${ }^{3}$ Department of Pathology, University of Southern \\ California, Los Angeles, California; and ${ }^{4}$ Vasgene Therapeutics Inc., Los Angeles, California
}

Accumulating evidence suggests that ephrin type $B$ receptor 4 (EphB4) plays a key role in the progression of numerous cancer types. In this study, we developed a series of ${ }^{64} \mathrm{Cu}$-labeled antibodies for PET imaging of tumor EphB4 expression. Methods: Anti-EphB4 antibodies (hAb47 and hAb131) were conjugated with the ${ }^{64} \mathrm{Cu}$-chelator DOTA through lysine, cysteine, or oligosaccharide on the antibody. DOTA-human IgG (hlgG) was also prepared as a control, which did not bind to EphB4. The EphB4 binding activity of these probes was evaluated through the bead-based binding assay with EphB4-alkaline phosphatase. The resulting PET probes were further evaluated in both HT29 (colorectal cancer) and MDAMB-231 (breast cancer) xenografts. Results: All 3 conjugation methods retained most of the EphB4 binding activity of the antibodies $(83.85 \% \pm 3.82 \%, 76.25 \% \pm 5.90 \%, 98.93 \% \pm 3.75 \%$, and $82.09 \% \pm 4.14 \%$ for DOTA-Lys-hAb47, DOTA-Cys-hAb47, DOTASug-hAb47, and DOTA-Lys-hAb131, respectively). Although DOTASug-hAb47 demonstrated the highest receptor binding activity based on a EphB4 binding assay, the corresponding PET probe was trapped in the liver quickly in vivo. In HT29 xenografts, both ${ }^{64}$ Cu-DOTA-Lys-hAb47 and ${ }^{64}$ Cu-DOTA-Cys-hAb47 demonstrated prominent tumor accumulation, which reached a maximum at $48 \mathrm{~h}$ after injection (18.13 \pm 1.73 percentage injected dose [\%ID]/g and $11.81 \pm 2.05 \% \mathrm{ID} / \mathrm{g}$, respectively). In contrast, ${ }^{64} \mathrm{Cu}$-DOTA-LyshlgG had a low tumor accumulation, thus demonstrating the target specificity of EphB4-antibody-based probes. Moreover, ${ }^{64} \mathrm{Cu}-$ DOTA-Lys-hAb131 (29.48 $\pm 2.60 \%$ ID/g) demonstrated significantly higher HT29 tumor accumulation than ${ }^{64} \mathrm{Cu}-\mathrm{DOTA}-\mathrm{Lys}-\mathrm{hAb} 47$. ${ }^{64} \mathrm{Cu}$-DOTA-Lys-hAb131 was also found to specifically accumulate in the MDA-MB-231 tumor model $(12.96 \pm 2.31 \% \mathrm{ID} / \mathrm{g})$. Conclusion: We have demonstrated that EphB4 can serve as a valid target for colorectal and breast cancer imaging. This approach would be valuable for evaluating disease course and therapeutic efficacy at the earliest stages of anti-EphB4 treatment. Moreover, these newly developed probes may have important applications in other cancer types overexpressing EphB4.

Received Nov. 5, 2012; revision accepted Jan. 22, 2013.

For correspondence or reprints contact either of the following:

Zibo Li, Molecular Imaging Center, Department of Radiology, University of

Southern California, Los Angeles, CA 90033.

E-mail: ziboli@usc.edu

Hong Shan, Department of Radiology, Third Affiliated Hospital of Sun YatSen University, Guangzhou, China 510630.

E-mail: shanhong@mail.sysu.edu.cn

${ }^{*}$ Contributed equally to this work.

Published online May 10, 2013.

COPYRIGHT (C) 2013 by the Society of Nuclear Medicine and Molecular Imaging, Inc.
Key Words: EphB4; breast cancer; colorectal cancer; ${ }^{64} \mathrm{Cu}$; PET

J Nucl Med 2013; 54:1094-1100

DOI: 10.2967/jnumed.112.116822

C olorectal cancer is one of the leading causes of cancer-related death for both men and women in the United States, with estimated new cancer cases and deaths in 2012 numbering 143,460 and 51,690, respectively (1). Colorectal cancer mortality has remained high over the last $20 \mathrm{y}$ because nearly half the cases were not detected until the colorectal cancer had advanced to a later, incurable, stage. Breast cancer is the most frequently diagnosed cancer for women in North America. The prognosis is favorable for women with clinically confined tumors at the time of diagnosis, but mortality rates are greater than $80 \%$ when the tumor has metastasized to distant sites (2). There is clearly a need to develop new diagnostic and therapeutic methods for both colorectal cancer and metastatic breast cancer. Recent advances in tumor biology allow for a more complete understanding of genetic and epigenetic changes in cancer development, metastasis, and progression, thus providing unprecedented opportunities for the development of noninvasive molecular imaging technologies for early detection and treatment monitoring of colorectal cancer and breast cancer (3).

Ephrin and its receptors are the largest subfamily of receptor tyrosine kinases. Ephrin receptors are divided into 2 subgroups: EphA and EphB. EphB4, in particular, has been found to play an important role in cell aggregation and migration, neural development, embryogenesis, angiogenesis, and vascular development (4-6). Not surprisingly, EphB4 was reported to be overexpressed in many human tumor tissues such as breast (7), colon $(8-10)$, bladder $(11)$, prostate $(12,13)$, and ovary $(14,15)$. Because of the extremely important function and widespread expression of EphB4 in colorectal cancer and breast cancer, therapies focusing on this target have become potentially important components of cancer treatment strategies $(7,8,16)$. Therefore, an efficient imaging modality, such as PET or SPECT, coupled with suitably radiolabeled EphB4 antibodies or EphB4 binding peptides, will provide important information for assessing EphB4-targeted therapy. Furthermore, the development of these novel imaging probes could lead to novel diagnostic methods for colorectal cancer and breast cancer, help us better predict which patients and individual tumors are likely to 
respond to novel interventions targeting EphB4, and make it possible to directly monitor the responses toward therapeutic interventions.

Recently, EphB4-specific mouse-originated monoclonal antibodies have been developed to target the extracellular domain of EphB4, which did not recognize other members of the ephrin family (17). One of these humanized antibodies, hAb47, binds to fibronectinlike domain 2 of both human and murine EphB4 with high affinity $(0.8 \mathrm{nmol} / \mathrm{L})$. The other humanized antibody, hAb131, binds specifically to human EphB4 with similar binding affinity $(1.0 \mathrm{nmol} / \mathrm{L})$. In this study, we investigated whether anti-EphB4 antibodies can be used for noninvasive imaging of EphB4 after modification with 3 different methods.

\section{MATERIALS AND METHODS}

Unless noted otherwise, all chemicals were of analytic grade and purchased from Sigma-Aldrich. DOTA analogs were purchased from Macrocyclics, Inc. Size-exclusion PD-10 columns were purchased from GE Healthcare. Monoclonal antibodies to EphB4 and EphB4-alkaline phosphatase were kindly provided by Vasgene Therapeutics, Inc. Human IgG (hIgG) was purchased from Rockland. CD31 antibody was from BD Pharmingen. $\beta$-actin antibody was from Sigma-Aldrich. The secondary antibodies goat antihuman Alexa Fluor 568 and goat antirat Alexa Fluor 488 were from Invitrogen. ${ }^{64} \mathrm{Cu}$ was obtained from Washington University and the University of Wisconsin. ${ }^{64} \mathrm{Cu}$ was produced using the ${ }^{64} \mathrm{Ni}(\mathrm{p}, \mathrm{n}){ }^{64} \mathrm{Cu}$ nuclear reaction and supplied in high specific activity as ${ }^{64} \mathrm{CuCl}_{2}$ in $0.1 \mathrm{~N} \mathrm{HCl}$.

\section{Chemistry and Radiochemistry}

Amino groups on the lysine side chain of hIgG, hAb47, and hAb131 were conjugated with DOTA (molar ratio, 1:20) to form DOTA-Lys-hIgG, DOTA-Lys-hAb47, and DOTA-Lys-hAb131, respectively. In brief, DOTA$N$-hydroxysulfosuccinimidyl was synthesized in situ as reported previously (18). Without purification, DOTA- $N$-hydroxysulfosuccinimidyl was cooled to $4^{\circ} \mathrm{C}$ and added to the antibodies/hIgG at $\mathrm{pH} 8.5$ ( $0.1 \mathrm{M}$ borate buffer). The reaction mixture was incubated at $4^{\circ} \mathrm{C}$ overnight. The DOTA conjugated antibodies/hIgG were then purified using a PD-10 column and concentrated by a Centricon filter (Millipore). The final concentration measurement was based on ultraviolet absorbance at $280 \mathrm{~nm}$ using unconjugated antibody of known concentrations as a standard.

To synthesize DOTA-Cys-hAb47, an aqueous solution of hAb47 (500 $\mu \mathrm{g}$ ) was mixed with $5 \mu \mathrm{L}$ of $100 \mathrm{mM}$ tris(2-carboxyethyl)phosphine in phosphate-buffered saline (PBS) for $1 \mathrm{~h}$. Maleimido-monoamideDOTA was added to the reaction mixture in a molar ratio of 1:20. After $30 \mathrm{~min}$, the final product (DOTA-Cys-hAb47) was purified by a PD-10 column and concentrated by a Centricon filter. The final concentration measurement was based on ultraviolet absorbance at $280 \mathrm{~nm}$ using an unconjugated antibody of known concentrations as a standard.

hAb47 $(500 \mu \mathrm{g})$ was dissolved in $10 \mathrm{mM}$ freshly prepared sodium periodate solution $(200 \mu \mathrm{L})$ in sodium acetate buffer $(\mathrm{pH} 5.5)$. After $20 \mathrm{~min}$, the oxidation product, Sug-hAb47, was purified by a PD-10 column. DOTA-hydrazide was prepared in situ by mixing DOTA-NHS and adipic acid dihydrazide at room temperature for $1 \mathrm{~h}$ with $1: 2$ reaction ratio in borate buffer $(\mathrm{pH} 8.5)$. Without purification, 20 equivalents of DOTA-hydrazide were added to Sug-hAb47 at pH 7 (phosphate buffer). After $5 \mathrm{~h}$ of incubation, the conjugate, DOTASug-hAb47, was purified by a PD-10 column and concentrated by a Centricon filter. The final concentration measurement was based on ultraviolet absorbance at $280 \mathrm{~nm}$ using an unconjugated antibody of known concentrations as a standard. The isotopic dilution method was used to determine the DOTA number for each conjugate (Supplemental Table 1; supplemental materials are available online at http:// jnm.snmjournals.org).
All ${ }^{64} \mathrm{Cu}$ labeling reactions were performed using the same protocol. In brief, $25-50 \mu \mathrm{g}$ of the antibodies were loaded to ${ }^{64} \mathrm{CuCl}_{2}$ (37-74 $\mathrm{MBq}{ }^{64} \mathrm{Cu}$ per $25 \mu \mathrm{g}$ antibody). The reaction mixture was incubated for $1 \mathrm{~h}$ at $40^{\circ} \mathrm{C}$ with constant shaking. The ${ }^{64} \mathrm{Cu}$-labeled antibody conjugates were then purified by a PD-10 column using $\times 1$ PBS as the mobile phase. The radioactive fraction containing ${ }^{64} \mathrm{Cu}$-DOTA product was collected for further in vitro and in vivo experiments.

\section{Binding Activity Assay}

The EphB4 binding affinity of DOTA-Lys-hIgG, DOTA-Lys-hAb47, DOTA-Lys-hAb131, DOTA-Cys-hAb47, and DOTA-Sug-hAb47 was evaluated through the bead-based binding assay with EphB4-alkaline phosphatase. EphB4-alkaline phosphatase was incubated with each of these immobilized on protein G-agarose $(5 \mu \mathrm{L} /$ tube) for $1 \mathrm{~h}$. Unbound proteins were removed by washing twice with PBS, and precipitated alkaline phosphatase activity was detected with para-nitrophenylphosphate. The EphB4 binding activity of unmodified hAb47/hAb131 and hIgG was also evaluated as a positive and negative control, respectively.

\section{Small-Animal PET and Image Analysis}

Human breast cancer cell lines (MDA-MB-231) and human colorectal cancer cell lines (HT29) were obtained from American Type Culture Collection and maintained under standard conditions. All animal experiments were performed under a protocol approved by the University of Southern California Institutional Animal Care and Use Committee. Tumor models were established in 4- to 6-wk-old female athymic nude mice obtained from Harlan. For the colorectal tumor model, $2 \times 10^{6}$ HT29 cells suspended in $100 \mu \mathrm{L}$ of PBS were injected into the front flank of the mice subcutaneously. For the breast cancer orthotopic model, $2 \times 10^{6}$ MDA-MB-231 cells suspended in $100 \mu \mathrm{L}$ of PBS were injected into the mammary fat pad subcutaneously. The mice were used for smallanimal PET imaging studies when the tumor volume reached $0.4-0.6 \mathrm{~cm}$ in diameter (2-4 wk after inoculation of the cancer cells).

PET scanning and image analysis were performed using a microPET R4 rodent model scanner (Siemens Medical Solutions) as previously reported $(19,20)$. A 3.7-7.4 MBq dose of PET probe was intravenously injected into each mouse under isoflurane anesthesia. Static scans were acquired at 1,24 , and $48 \mathrm{~h}$ after injection. The images were reconstructed by 2-dimensional ordered-subsets expectation maximum. For each small-animal PET scan, regions of interest were drawn over the tumor, normal tissue, and major organs on decaycorrected whole-body coronal images. The radioactivity concentration (accumulation) within a tumor was obtained from the mean value within the multiple regions of interest and then converted to percentage injected dose (\%ID)/g (21). For both HT29 and MDA-MB-231 tumor models, ${ }^{64} \mathrm{Cu}$-DOTA-hIgG was used as the control to confirm the receptor specificity of ${ }^{64} \mathrm{Cu}$-labeled anti-EphB4 antibody.

\section{Sodium Dodecyl Sulfate Polyacrylamide Gel Electrophoresis (SDS-PAGE) and Western Blotting}

Three micrograms of hAb47, DOTA-lys-hAb47, DOTA-cys-hAb47, or DOTA-Sug-hAb47 were mixed with Laemmle buffer (BioRad) without dithiothreitol and heated at $70^{\circ} \mathrm{C}$ for $10 \mathrm{~min}$. The samples were fractionated on a 4\%-20\% Tris-glycine gradient gel (Bio-Rad). Then, the gel was stained with Coomassie blue and scanned in an 800$\mathrm{nm}$ channel with an Odyssey infrared imager (LI-COR).

Proteins extracted from tumor cells were resolved on 4\%-20\% SDS-PAGE and transferred onto polyvinylidene difluoride membrane (Bio-Rad). Nonspecific binding was blocked with 5\% nonfat dry milk in Tris-buffered saline with $0.1 \%$ Tween-20. Membranes were first probed with primary antibody overnight at $4{ }^{\circ} \mathrm{C}$, washed and probed with the IRDye 800 secondary antibody for $1 \mathrm{~h}$ at room temperature, and scanned with an Odyssey infrared imager. 


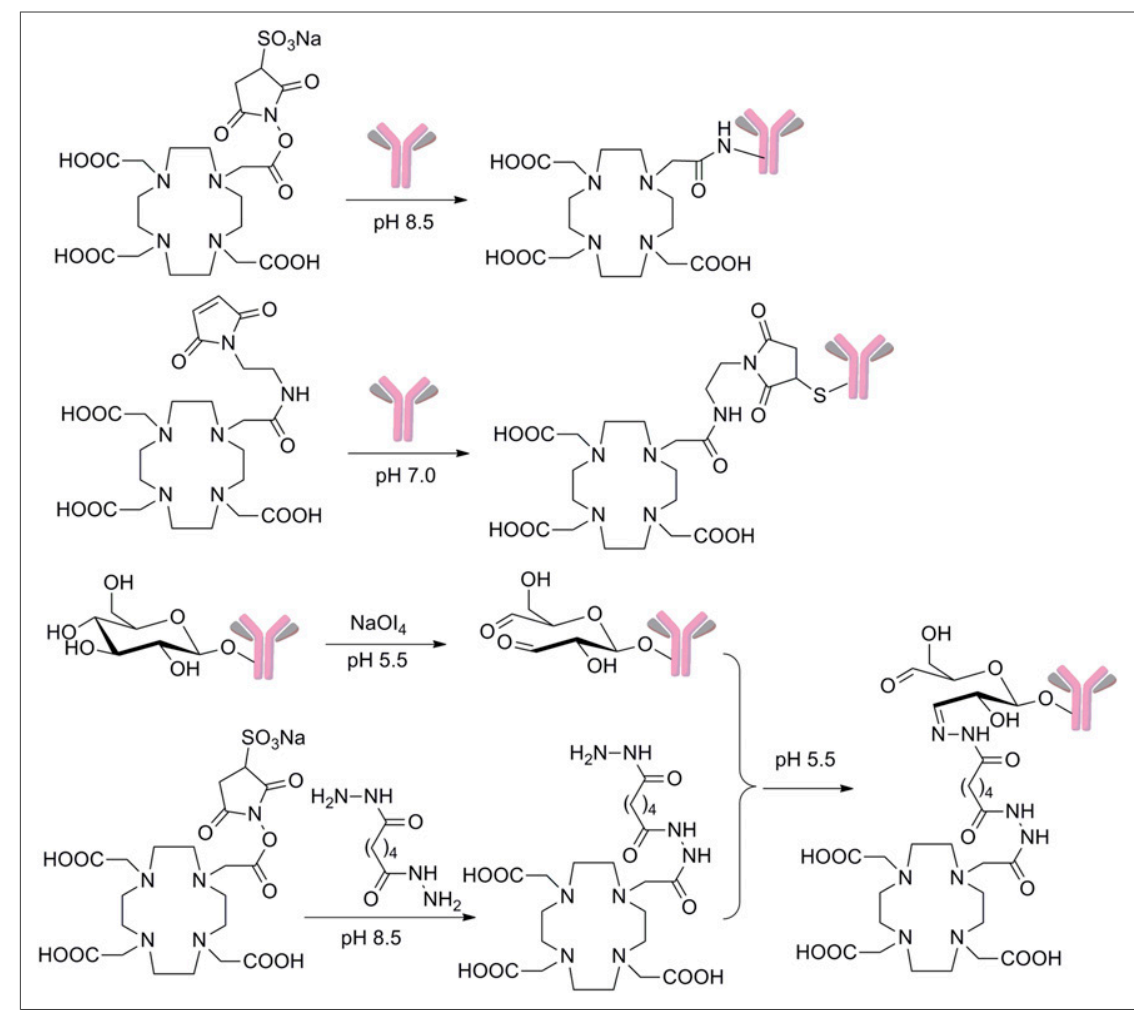

FIGURE 1. Schematic illustration of antibody conjugation with DOTA via amino group, sulfhydryl group on cysteine side chain, and aldehyde-dihydrazide coupling after oxidation of sugar moiety on antibody by sodium periodate.

\section{Immunofluorescence Staining}

For the antibody distribution assay, $30 \mu \mathrm{g}$ of DOTA-lys-hIgG, DOTA-lys-hAb47, or DOTA-lys-hAb131 were injected via the tail vein into each mouse bearing HT29 tumor. At $48 \mathrm{~h}$ after injection, the mice were euthanized and the tumors dissected. Frozen sections of tumors $(5 \mu \mathrm{m})$ were fixed in $4 \%$ paraformaldehyde (Electron Microscopy Sciences) and blocked with $10 \%$ normal goat serum (Invitrogen). Sections were then incubated with anti-CD31 primary antibody overnight at $4^{\circ} \mathrm{C}$, followed by the corresponding secondary antibody for $1 \mathrm{~h}$ at room temperature. Subsequently, the slides were covered with Vectashield mounting medium (Vector Labs) with 4,6-diamino-2-phenylindole and images were obtained with an Eclipse 80i fluorescence microscope (Nikon). Secondary antibody goat antihuman Alexa Fluor 568 and goat antirat Alexa Fluor 488 were used to detect antibodies (hIgG, hAb47, or hAb131) and $\mathrm{CD} 31$, respectively.

\section{Statistical Analysis}

Quantitative data are expressed as mean \pm SD. Means were compared using 1-way ANOVA and the Student $t$ test. $P$ values of less than 0.05 were considered statistically significant.

\section{RESULTS}

\section{Chemistry and Radiochemistry}

hAb47 was conjugated with ${ }^{64} \mathrm{Cu}$ chelator through 3 methods. Each hAb47 molecule has 4 terminal amino groups and several lysine side chain amino groups available for conjugation with DOTA- $N$-hydroxysulfosuccinimidyl, which would lead to DOTALys-hAb47. DOTA-Lys-hIgG and DOTA-Lys-hAb131 were prepared using the same procedure. Alternatively, after partial reduction of disulfide bonds, free sulfhydryl groups of hAb47 molecule were available for conjugation with DOTA-maleimide, resulting in DOTA-Mal-hAb47. The third conjugation method was performed through the conjugation with oxidized oligosaccharide, which resulted in DOTA-Sug-hAb47 (Fig. 1). The number of DOTA per antibody was determined to be between $0.93 \pm$ 0.18 and $3.58 \pm 0.34$ (Supplemental Table 1). Fast protein liquid chromatography analysis found no apparent aggregation after DOTA conjugation of the antibodies (Supplemental Figs. 1-3). The radiolabeling step took approximately 90 min for each antibody, and the radiochemical yields for ${ }^{64} \mathrm{Cu}$-DOTA-Lys-hIgG, ${ }^{64} \mathrm{Cu}$-DOTA-Lys-hAb47, ${ }^{64} \mathrm{Cu}$-DOTA-LyshAb131, ${ }^{64} \mathrm{Cu}-\mathrm{DOTA}-\mathrm{Cys}-\mathrm{hAb} 47$, and ${ }^{64} \mathrm{Cu}$-DOTA-Sug-hAb47 were $65 \%, 74 \%$, $78 \%, 85 \%$, and $80 \%$, respectively.

\section{Binding Activity of DOTA Monoclonal Antibodies to EphB4}

To study the impact of DOTA conjugation on EphB4 binding ability, a binding activity assay was performed. After modification, DOTA-Lys-hAb47 and DOTACys-hAb47 had comparable EphB4 binding activities $(83.85 \% \pm 3.82 \%$ and $76.25 \% \pm$ $5.90 \%$, respectively). In contrast, DOTASug-hAb47 maintained $98.93 \% \pm 3.75 \%$ EphB4 binding activity and DOTA-Lys-hIgG had minimal binding toward this target. Moreover, 82.09\% $\pm 4.14 \%$ EphB4 binding affinity was maintained for DOTA-Lys-hAb131, which binds to the fibronectinlike domain 2 of EphB4 receptor. The binding assay showed that DOTA-Sug-hAb47 provides significantly higher binding affinity than do DOTA-Lys-hAb47 and DOTA-Cys-hAb47 $(P<$ 0.05) (Fig. 2A). The molecular weight study showed that DOTACys-hAb47 via the reduction of disulfide bonds broke into the unique parts of the antibody whereas DOTA conjugation via lysines and sugar oxidation kept the antibody intact (Fig. 2B).

\section{EphB4 Expression on Cell Lines}

Measured by Western blotting, EphB4 expression levels were determined in a human breast cancer cell line (MDA-MB-231) and a human colorectal cancer cell line (HT29). Among these 2 cell lines, HT29 demonstrated the higher EphB4 expression (Figs. 2C and D). Immunofluorescence EphB4/CD31 staining revealed that EphB4 expression was prominent on HT29 tumor, as compared with MDA-MB-231 (Supplemental Fig. 4). In this study, HT29 and MDA-MB-231 tumor xenografts were both used for the following in vivo evaluation of ${ }^{64} \mathrm{Cu}$-labeled antibodies to test the relationship between EphB4 expression and corresponding tumor uptake.

\section{Small-Animal PET Imaging of EphB4}

The tumor targeting efficacy of ${ }^{64} \mathrm{Cu}$-labeled hAb47 was evaluated by multiple-time-point static small-animal PET scans in HT29 tumor-bearing mice ( $n=3 /$ tracer). Representative decaycorrected coronal small-animal PET images at different time points after injection are shown in Figure 3. Although DOTA- 


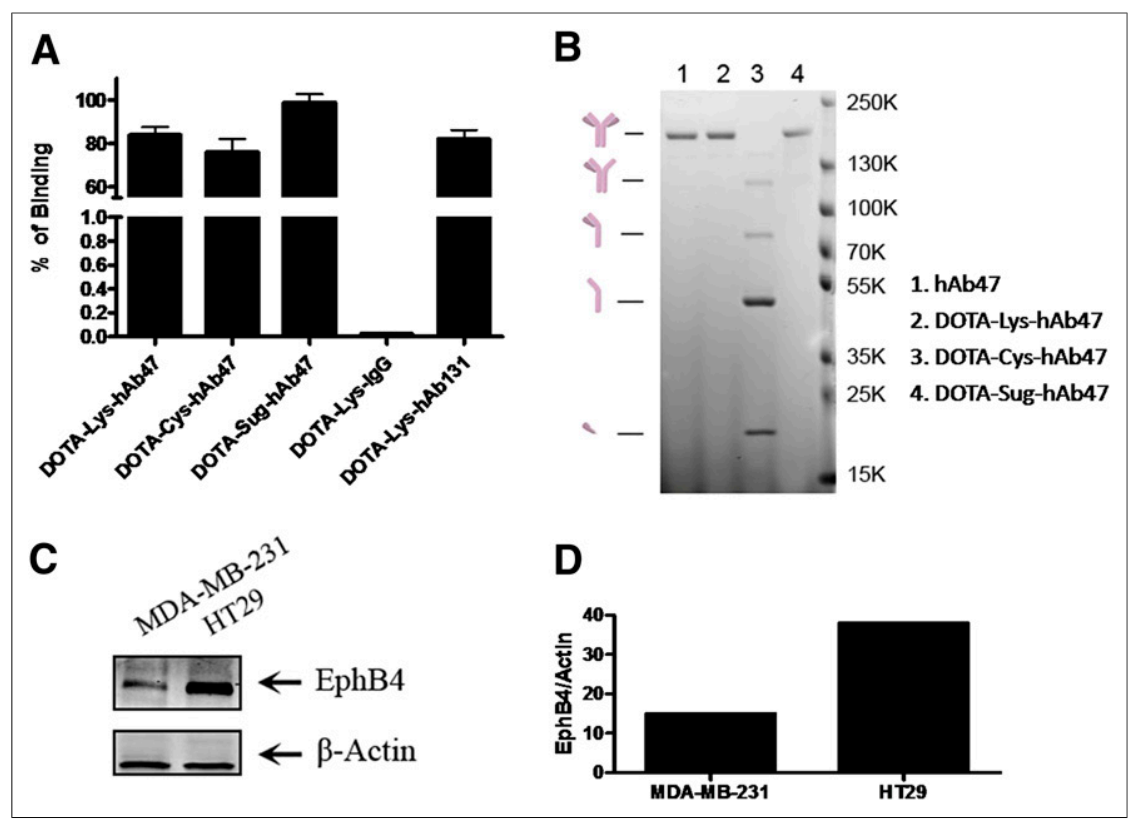

FIGURE 2. (A) Binding activity of manipulated hAb47 and hAb131 antibodies to EphB4 receptor. (B) Molecular weight study of DOTA-conjugated hAb47 by SDS-PAGE. Positions of molecular weight markers are given on right. Position of intact hAb47 is shown on left. (C) EphB4 protein levels were determined by Western blotting in MDA-MB-231 and HT29 cell lines. (D) Ratios of EphB4 concentration to $\beta$-actin in MDA-MB-231 and HT29 cell lines.

Sug-hAb47 demonstrated the highest EphB4 binding affinity in vitro, ${ }^{64} \mathrm{Cu}$-DOTA-Sug-hAb47 was quickly trapped in mouse liver in vivo, significantly reducing the amount of probe circulating in the blood. In fact, tumor uptake of ${ }^{64} \mathrm{Cu}$-DOTA-Sug-hAb47 was only $3.89 \pm 0.72,7.60 \pm 0.79$, and $9.54 \pm 0.59 \% \mathrm{ID} / \mathrm{g}$ at 1,24 , and $48 \mathrm{~h}$ after injection. At $1 \mathrm{~h}$ after injection, both ${ }^{64} \mathrm{Cu}$-DOTALys-hAb47 and ${ }^{64} \mathrm{Cu}-\mathrm{DOTA}-\mathrm{Cys}-\mathrm{hAb} 47$ were circulating mainly in the blood pool. The tumors were clearly visualized, with high tumor-to-background contrast at late time points. The activity accumulation of ${ }^{64} \mathrm{Cu}-\mathrm{DOTA}-L y s-h A b 47$ (18.13 $\pm 1.73 \% \mathrm{ID} / \mathrm{g} 48 \mathrm{~h}$ after injection) in HT29 tumor was significantly higher $(P=$ $0.015)$ than that of ${ }^{64} \mathrm{Cu}$-DOTA-Cys-hAb47 $(11.81 \pm 2.05$ $\% \mathrm{ID} / \mathrm{g})$. No significant difference in liver $(P=0.100)$ or kidney $(P=0.661)$ uptake was observed between these 2 probes. On the basis of these results, we focused on Lys modification in the following studies.

Development of ${ }^{64} \mathrm{Cu}$-DOTA-Lys-hAb131 was based on an antibody that will internalize after binding to the receptor. In contrast, hAb47 does not internalize after binding to the receptor (17). In the HT29 tumor model, ${ }^{64} \mathrm{Cu}$-DOTA-Lys-hAb131 demonstrated significantly higher tumor uptake $(21.90 \pm 1.93$ and $29.48 \pm 2.60 \% \mathrm{ID} / \mathrm{g}$ at 24 and $48 \mathrm{~h}$ after injection, respectively) than did ${ }^{64} \mathrm{Cu}$-DOTALys-hAb47 $(16.00 \pm 1.88$ and $18.13 \pm 1.73 \% \mathrm{ID} / \mathrm{g}$ at 24 and $48 \mathrm{~h}$ after injection, respectively; $P=0.019$ and 0.003 , respectively) (Figs. 4 and 5). ${ }^{64} \mathrm{Cu}$-DOTA-Lys-hAb131 was also evaluated in the MDA-MB-231 breast cancer model (Fig. 4), with uptake of $6.56 \pm 1.14,10.07 \pm 1.77$, and $12.96 \pm 2.31 \% \mathrm{ID} / \mathrm{g}$ at 1,24 , and $48 \mathrm{~h}$ after injection, respectively. To confirm the target specificity of ${ }^{64} \mathrm{Cu}$-labeled hAb47 and hA131, ${ }^{64} \mathrm{Cu}$-DOTA-Lys-hIgG was prepared and imaged in HT29 and MDA-MB-231 tumor models. Without EphB4-targeting capability, ${ }^{64} \mathrm{Cu}$-DOTA-Lys-hIgG demonstrated $6.83 \pm 2.09 \% \mathrm{ID} / \mathrm{g}$ uptake in HT29 tumor and $6.92 \pm 1.07$
$\%$ ID/g uptake in MDA-MD-231 tumor at $48 \mathrm{~h}$ after injection-a result that could be due mainly to passive targeting.

\section{Immunofluorescence Staining}

To further confirm that hAb47 and hAb131 targeted the HT29 tumor cells in vivo, we stained the tumor tissue using intravenously injected hAb47 and hAb131 as the primary antibody and goat antihuman antibody conjugated with Alexa Fluor as the secondary antibody. Images were obtained under identical conditions and displayed at the same scale so that the relative brightness observed in the images reflected the difference in relative $\mathrm{hAb} 47$ and hAb131 concentration level (Fig. 6). hIgG was used as a control. hAb131 demonstrated higher targeting efficiency than hAb47-a result that could probably be caused by a different targeting site on EphB4. The targeting site of mAb131 is at the upper site of the transmembrane receptor; presumably, mAb131 has a better chance to interact with EphB4 because of the much lower steric hindrance (Supplemental Fig. 5). The negative control hIgG showed minimal distribution in HT29 tumor, clearly demonstrating the target specificity of EphB4-targeted probes. Vessels and macrophages are important components besides tumor cells in tumor tissues $(22,23)$. Therefore, colocalization of antibodies and vessels or macrophages could help better elucidate the antibody distribution pattern in tumor tissues. CD31 is a marker of both vascular endothelial cells and macrophages $(24,25)$. The absence of overlap between antibodies (hIgG, hAb47, or hAb131) and CD31 shown in Figure 6 further demonstrates that hAb47 and hAb131 bind specifically to HT29 tumor cells.

\section{DISCUSSION}

Accumulating evidence suggests that EphB4 plays a key role in the progression of numerous cancer types. In fact, therapies focusing on EphB4 have become potentially important components of strategies for treating various types of cancer, including colorectal cancer and breast cancer. The ability to image EphB4 in vivo could therefore be valuable for the sensitive detection of lesions, prognostic evaluation, selection of patients for clinical trials, better treatment monitoring and dose optimization, and elucidation of the mechanisms of treatment efficacy underlying the relevant signaling pathways (26).

Previously, the development of peptide-based probes for EphB4targeted imaging has been based on 12-mer peptides identified by phage display technology (27-29). However, both absolute tumor uptake and the pharmacokinetics of these probes need to be further improved. Antibodies are considered as potentially the most specific probes for imaging because they offer an unmatched ability to bind selectively to any target (30). Recently, anti-EphB4 antibodies have been obtained for EphB4 binding with $1 \mathrm{nM}$ affinity. Because the antibody has much higher EphB4 binding affinity and specificity than does small peptide, increased tumor uptake may result. Moreover, the antibody-based imaging probes would be more suitable for 


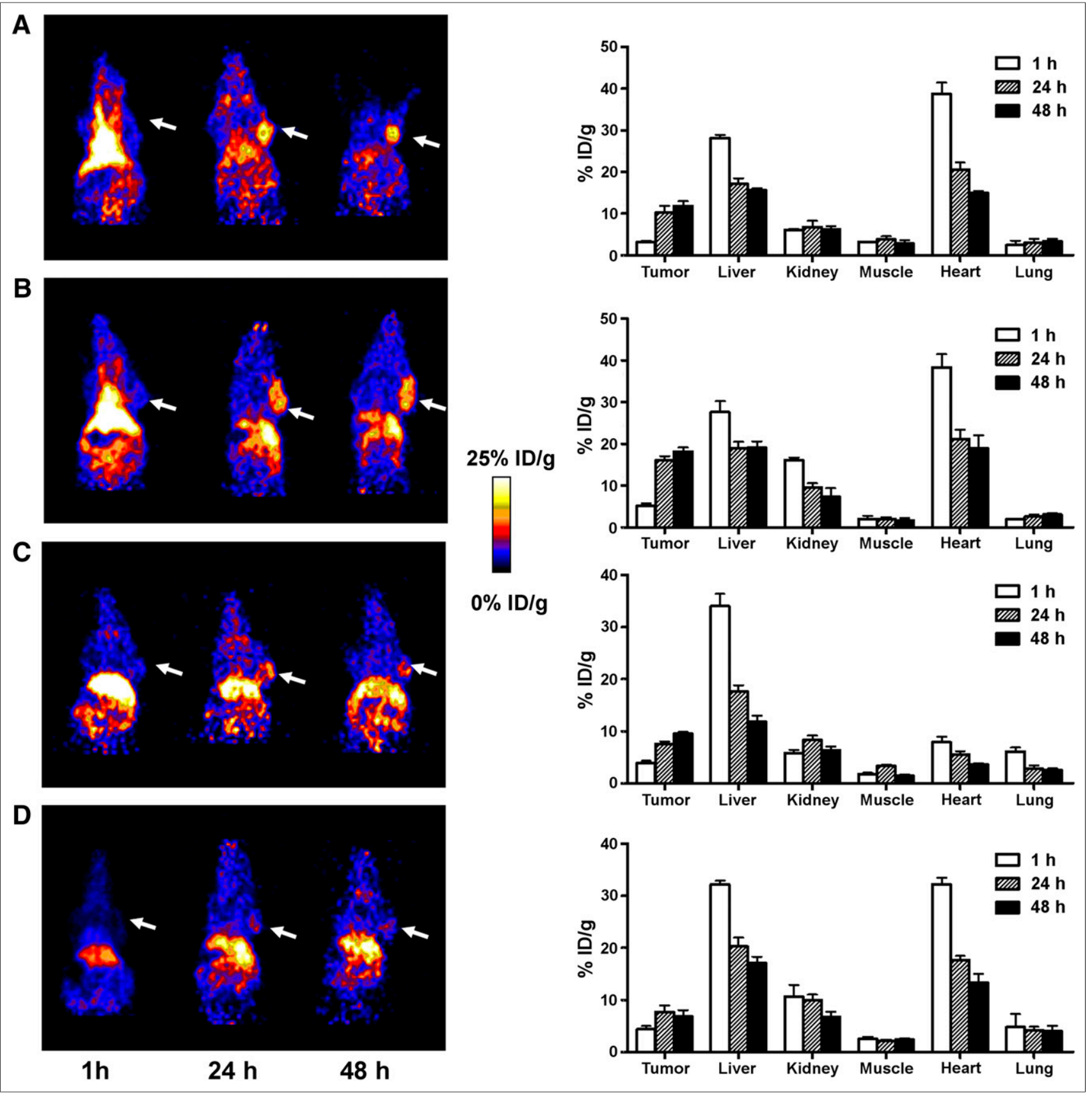

FIGURE 3. (Left) Decay-corrected whole-body coronal small-animal PET static scans of mice bearing HT29 tumors. Images were obtained at 1, 24, and $48 \mathrm{~h}$ after injection of ${ }^{64} \mathrm{Cu}-\mathrm{DOTA}-\mathrm{Cys}-\mathrm{hAb} 47$ (A), ${ }^{64} \mathrm{Cu}-\mathrm{DOTA}-\mathrm{Lys}-\mathrm{hAb} 47$ (B), ${ }^{64} \mathrm{Cu}-\mathrm{DOTA}-\mathrm{Sug}-\mathrm{hAb} 47$ (C), and ${ }^{64} \mathrm{Cu}-\mathrm{DOTA}$-Lys-hlgG (D). Tumors are indicated by arrows. (Right) Quantification of radioactivity in major organs.

patient screening as they are closely related to the antibody drug to be used in immunotherapy. In this study, we developed a series of EphB4-targeted PET probes based on 2 anti-EphB4 monoclonal antibodies, hAb47 and hAb131 (17).

In the initial study, hAb47 was conjugated with DOTA chelator through 3 different methods using the amino groups on the lysine side chain, the sulfhydryl group on cysteine after partial reduction of disulfide bonds, and the aldehyde groups after oxidation of oligosaccharide. SDS-PAGE showed that amino modification and oligosaccharide oxidation have a minimal effect on the integrity of the antibody, whereas cysteine modification has broken the antibody into small parts through the use of tris(2-carboxyethyl)phosphine (Fig. 2B). The EphB4 binding assay showed that modifications through lysine (DOTA-Lys-hAb47) and cysteine (DOTA-Cys-hAb47) reduced the target binding affinity by only around $20 \%$ when the reaction conditions were controlled carefully. As expected, we did not observe an obvious reduction in EphB4 binding when the conjugation was performed at the oligosaccharide site (DOTA-Sug-hAb47). A high radiolabeling yield was obtained for antibodies modified through all 3 conjugation methods. With these promising results at hand, we further tested the PET probes in a HT29 tumor model. Although DOTA-Sug-hAb47 had the highest EphB4 binding affinity, we were 


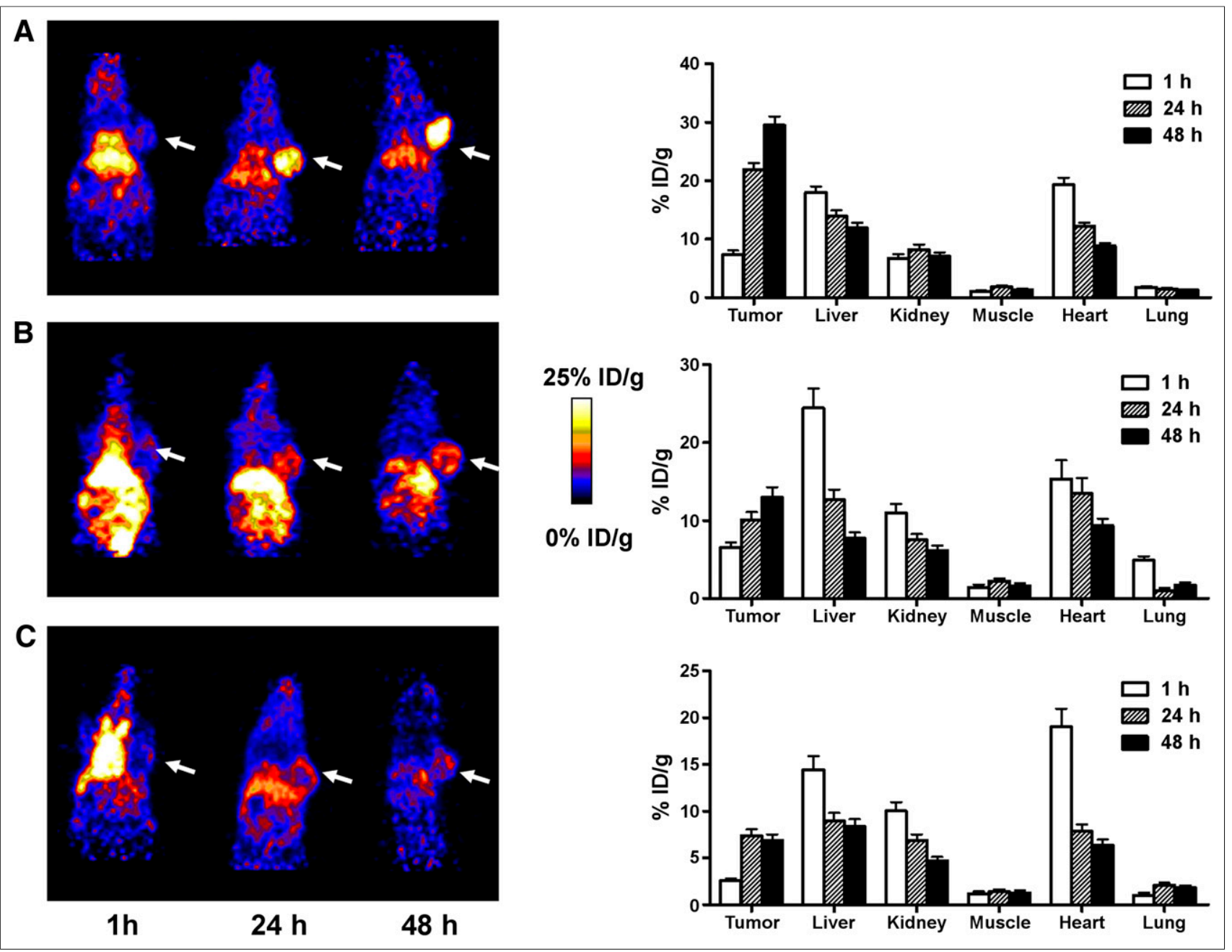

FIGURE 4. (Left) Decay-corrected whole-body coronal small-animal PET static scans of mice bearing HT29 tumors (A) or MDA-MB-231 tumors (B and C). Images were obtained at 1, 24, and $48 \mathrm{~h}$ after injection of ${ }^{64} \mathrm{Cu}-\mathrm{DOTA}-\mathrm{Lys}-\mathrm{hAb} 131(\mathrm{~A}),{ }^{64} \mathrm{Cu}-\mathrm{DOTA}-\mathrm{Lys}-\mathrm{hAb} 131$ (B), and ${ }^{64} \mathrm{Cu}-\mathrm{DOTA}-\mathrm{Lys}-\mathrm{hlgG}(\mathrm{C})$. Tumors are indicated by arrows. (Right) Quantification of radioactivity in major organs.

surprised to find that ${ }^{64} \mathrm{Cu}$-DOTA-Sug-hAb47 had a circulation profile very different from that of the other 2 probes (Supplemental Fig. 6). ${ }^{64} \mathrm{Cu}$-DOTA-Sug-hAb47 cleared quickly from the blood pool and had much lower uptake in HT29 tumors at all time points tested. In antibodies, glycosylation of the $\mathrm{CH}_{2}$ domain through the attachment of oligosaccharides at asparagine 297 is a unique feature. Structural studies indicate that the complex oligosaccharides at asparagine 297 in antibodies are sequestered between the polypeptide chains in the $\mathrm{CH} 2$ domain $(31,32)$. This characteristic could avoid rapid clearance of antibodies from the circulation by carbohydrate-specific recognition systems in hepatic and reticuloendothelial tissue (33-35). Presumably, the rapid clearance of DOTA-Sug-hAb47 from blood in this study may have been due to exposure of galactose residues from the $\mathrm{CH} 2$ domain of antibodies after DOTA conjugation. Both ${ }^{64} \mathrm{Cu}-\mathrm{DOTA}-$ Lys-hAb47 and ${ }^{64} \mathrm{Cu}-\mathrm{DOTA}-\mathrm{Cys}-\mathrm{hAb} 47$ demonstrated prominent HT2 9 tumor uptake at 24 and $48 \mathrm{~h}$ after injection. In our following studies, we focused mainly on ${ }^{64} \mathrm{Cu}$-DOTA-Lys-hAb47, for which the preparation procedure not only is relatively simple but also maintains the integrity of the antibody. For ${ }^{64} \mathrm{Cu}$-DOTA-Lys-hAb47, both hAb47-EphB4 interaction and the enhanced-permeability-and-retention effect could contribute to tumor uptake (36). To estimate the specific targeting attributed to antibody-antigen interaction,
${ }^{64} \mathrm{Cu}$-DOTA-Lys-hIgG was synthesized as the control (to evaluate passive targeting). Tumor uptake of ${ }^{64} \mathrm{Cu}$-DOTA-Lys-hIgG was comparatively low at all time points examined, thus demonstrating the targeted specificity of ${ }^{64} \mathrm{Cu}-\mathrm{DOTA}-\mathrm{Lys}-\mathrm{hAb} 47$.

In addition to being evaluated in the HT29 tumor model, ${ }^{64} \mathrm{Cu}-$ DOTA-Lys-hAb131 was also evaluated in MDA-MB-231 tumor

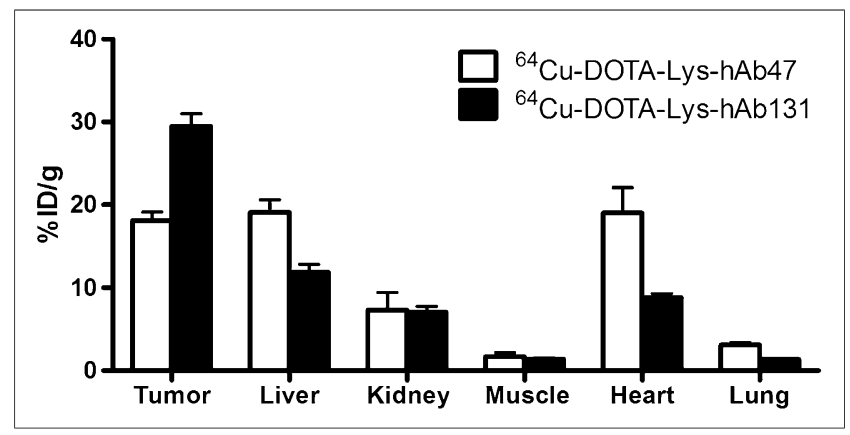

FIGURE 5. HT29 tumor and major organ uptake of ${ }^{64} \mathrm{Cu}$-DOTALys-hAb47 and ${ }^{64} \mathrm{Cu}$-DOTA-Lys-hAb131 at $48 \mathrm{~h}$ after injection. Data are expressed as average $\pm \operatorname{SD}(n=3)$. 


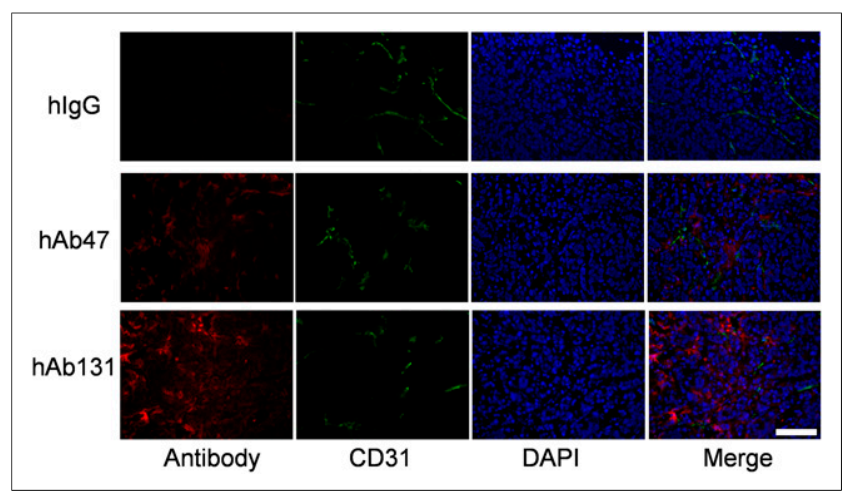

FIGURE 6. Immunofluorescence staining of antibodies and CD31 in HT29 tumor tissues. Images were obtained under identical conditions and displayed at same magnification and scale $(\times 200)$. Scale bar is $100 \mu \mathrm{m}$. DAPI $=4,6$-diamino-2-phenylindole.

(breast cancer). At 24 and $48 \mathrm{~h}$ after injection, ${ }^{64} \mathrm{Cu}$-DOTA-LyshAb131 demonstrated prominent tumor uptake significantly higher than that of ${ }^{64} \mathrm{Cu}$-DOTA-Lys-hIgG control. We also noticed that the MDA-MB-231 tumor had significantly lower tumor uptake than did HT29 tumor $(P<0.05)$, as could be caused by the difference in EphB4 expression level between these 2 tumor models (Fig. 2C). However, further investigation is needed to validate this statement since these 2 tumor models were established differently (subcutaneous vs. orthotopic).

\section{CONCLUSION}

We have described the in vitro and in vivo distribution of ${ }^{64} \mathrm{Cu}-$ labeled hAb47 and hAb131 antibodies in colorectal cancer and breast cancer models. This approach would be valuable for evaluating the pharmacokinetics, tumor-targeting efficacy, dose optimization, and dose interval of hAb47 and hAb131 antibody-based cancer therapeutics. Moreover, these newly developed probes might have important applications in other cancer types overexpressing EphB4.

\section{DISCLOSURE}

The costs of publication of this article were defrayed in part by the payment of page charges. Therefore, and solely to indicate this fact, this article is hereby marked "advertisement" in accordance with 18 USC section 1734 . This work was partially supported by the NIBIB (5R21EB012294-02), the Early (Margaret E.) Medical Research Trust, the American Cancer Society (121991-MRSG-12-034-01-CCE), the Department of Defense (BC102678), Vasgene Therapeutics Inc. (NIH: CA168158-01; CA171538-01, the National Natural Science Foundation of China (U1032002, 81071206), and Key Clinical Research Project 164 of the Public Health Ministry of China, 2010-2012. No other potential conflict of interest relevant to this article was reported.

\section{REFERENCES}

1. Siegel R, Naishadham D, Jemal A. Cancer statistics, 2012. CA Cancer J Clin. 2012;62:10-29.

2. Coleman RE, Smith P, Rubens RD. Clinical course and prognostic factors following bone recurrence from breast cancer. Br J Cancer. 1998;77:336-340.

3. Bombardieri E, Gianni L. The choice of the correct imaging modality in breast cancer management. Eur J Nucl Med Mol Imaging. 2004;31(suppl 1):S179-S186.

4. Dodelet VC, Pasquale EB. Eph receptors and ephrin ligands: embryogenesis to tumorigenesis. Oncogene. 2000;19:5614-5619.

5. Erber R, Eichelsbacher U, Powajbo V, et al. EphB4 controls blood vascular morphogenesis during postnatal angiogenesis. EMBO J. 2006;25:628-641.
6. Wang HU, Chen ZF, Anderson DJ. Molecular distinction and angiogenic interaction between embryonic arteries and veins revealed by ephrin-B2 and its receptor Eph-B4. Cell. 1998;93:741-753.

7. Kumar SR, Singh J, Xia G, et al. Receptor tyrosine kinase EphB4 is a survival factor in breast cancer. Am J Pathol. 2006;169:279-293.

8. Kumar SR, Scehnet JS, Ley EJ, et al. Preferential induction of EphB4 over EphB2 and its implication in colorectal cancer progression. Cancer Res. 2009;69:3736-3745.

9. Stephenson SA, Slomka S, Douglas EL, Hewett PJ, Hardingham JE. Receptor protein tyrosine kinase EphB4 is up-regulated in colon cancer. BMC Mol Biol. 2001;2:15.

10. Liu W, Ahmad SA, Jung YD, et al. Coexpression of ephrin-Bs and their receptors in colon carcinoma. Cancer. 2002;94:934-939.

11. Xia G, Kumar SR, Stein JP, et al. EphB4 receptor tyrosine kinase is expressed in bladder cancer and provides signals for cell survival. Oncogene. 2006;25:769-780.

12. Xia G, Kumar SR, Masood R, et al. EphB4 expression and biological significance in prostate cancer. Cancer Res. 2005;65:4623-4632.

13. Lee YC, Perren JR, Douglas EL, et al. Investigation of the expression of the EphB4 receptor tyrosine kinase in prostate carcinoma. BMC Cancer. 2005;5:119.

14. Castellano G, Reid JF, Alberti P, Carcangiu ML, Tomassetti A, Canevari S. New potential ligand-receptor signaling loops in ovarian cancer identified in multiple gene expression studies. Cancer Res. 2006;66:10709-10719.

15. Kumar SR, Masood R, Spannuth WA, et al. The receptor tyrosine kinase EphB4 is overexpressed in ovarian cancer, provides survival signals and predicts poor outcome. Br J Cancer. 2007;96:1083-1091.

16. Noren NK, Pasquale EB. Paradoxes of the EphB4 receptor in cancer. Cancer Res. 2007;67:3994-3997.

17. Krasnoperov V, Kumar SR, Ley E, et al. Novel EphB4 monoclonal antibodies modulate angiogenesis and inhibit tumor growth. Am J Pathol. 2010;176:2029-2038.

18. Li ZB, Cai W, Cao Q, et al. ${ }^{64} \mathrm{Cu}$-labeled tetrameric and octameric RGD peptides for small-animal PET of tumor $\alpha_{v} \beta_{3}$ integrin expression. J Nucl Med. 2007;48:1162-1171.

19. Liu S, Li D, Huang CW, et al. Efficient construction of PET/fluorescence probe based on sarcophagine cage: an opportunity to integrate diagnosis with treatment. Mol Imaging Biol. 2012;14:718-724.

20. Liu S, Li Z, Yap LP, Huang CW, Park R, Conti PS. Efficient preparation and biological evaluation of a novel multivalency bifunctional chelator for ${ }^{64} \mathrm{Cu}$ radiopharmaceuticals. Chemistry. 2011;17:10222-10225.

21. Wu Y, Zhang X, Xiong Z, et al. microPET imaging of glioma integrin $\alpha_{v} \beta_{3}$ expression using ${ }^{64} \mathrm{Cu}$-labeled tetrameric RGD peptide. J Nucl Med. 2005;46:1707-1718.

22. Weis SM, Cheresh DA. Tumor angiogenesis: molecular pathways and therapeutic targets. Nat Med. 2011;17:1359-1370.

23. Qian BZ, Pollard JW. Macrophage diversity enhances tumor progression and metastasis. Cell. 2010;141:39-51.

24. McKenney JK, Weiss SW, Folpe AL. CD31 expression in intratumoral macrophages: a potential diagnostic pitfall. Am J Surg Pathol. 2001;25:1167-1173.

25. Curat CA, Wegner V, Sengenes $C$, et al. Macrophages in human visceral adipose tissue: increased accumulation in obesity and a source of resistin and visfatin. Diabetologia. 2006;49:744-747.

26. Cai W, Rao J, Gambhir SS, Chen X. How molecular imaging is speeding up antiangiogenic drug development. Mol Cancer Ther. 2006;5:2624-2633.

27. Koolpe M, Burgess R, Dail M, Pasquale EB. EphB receptor-binding peptides identified by phage display enable design of an antagonist with ephrin-like affinity. J Biol Chem. 2005;280:17301-17311.

28. Xiong $\mathrm{C}$, Huang $\mathrm{M}$, Zhang R, et al. In vivo small-animal PET/CT of EphB4 receptors using ${ }^{64} \mathrm{Cu}$-labeled peptide. J Nucl Med. 2011;52:241-248.

29. Zhang R, Xiong C, Huang M, et al. Peptide-conjugated polymeric micellar nanoparticles for dual SPECT and optical imaging of EphB4 receptors in prostate cancer xenografts. Biomaterials. 2011;32:5872-5879.

30. Kaur S, Venktaraman G, Jain M, Senapati S, Garg PK, Batra SK. Recent trends in antibody-based oncologic imaging. Cancer Lett. 2012;315:97-111.

31. Deisenhofer J, Colman PM, Epp O, Huber R. Crystallographic structural studies of a human Fc fragment. II. A complete model based on a Fourier map at 3.5 A resolution. Hoppe Seylers Z Physiol Chem. 1976;357:1421-1434.

32. Silverton EW, Navia MA, Davies DR. Three-dimensional structure of an intact human immunoglobulin. Proc Natl Acad Sci USA. 1977;74:5140-5144.

33. Ashwell G, Morell AG. The role of surface carbohydrates in the hepatic recognition and transport of circulating glycoproteins. Adv Enzymol Relat Areas Mol Biol. 1974;41:99-128.

34. Stahl PD, Rodman JS, Miller MJ, Schlesinger PH. Evidence for receptor-mediated binding of glycoproteins, glycoconjugates, and lysosomal glycosidases by alveolar macrophages. Proc Natl Acad Sci USA. 1978;75:1399-1403.

35. Ashwell G, Harford J. Carbohydrate-specific receptors of the liver. Annu Rev Biochem. 1982;51:531-554.

36. Maeda H, Wu J, Sawa T, Matsumura Y, Hori K. Tumor vascular permeability and the EPR effect in macromolecular therapeutics: a review. J Control Release. 2000; 65:271-284. 